\title{
Expression, tissue localization and synergy of antimicrobial peptides and proteins in the immune response of the oyster Crassostrea gigas
}

\author{
Paulina Schmitt ${ }^{\mathrm{a}, \mathrm{b}, 1}$, Julien de Lorgeril ${ }^{\mathrm{a}}$, Yannick Gueguen ${ }^{\mathrm{a}, 2}$, Delphine Destoumieux-Garzón ${ }^{\mathrm{c}}$, \\ Evelyne Bachère ${ }^{a, *}$
}

\begin{abstract}
a Ifremer, UMR5119 “Écologie des Systèmes Marins Côtiers", Place Eugène Bataillon, 34095 Montpellier Cedex 5, France

b Université Montpellier 2, UMR5119 "Écologie des Systèmes Marins Côtiers", Place Eugène Bataillon, 34095 Montpellier Cedex 5, France

'CNRS, IRD and Université Montpellier 1, UMR5119 "Écologie des Systèmes Marins Côtiers", Place Eugène Bataillon, 34095 Montpellier Cedex 5, France

${ }^{1}$ Present address: Department of Pathology \& Laboratory Medicine, Keck School of Medicine of The University of Southern California, USC Norris Cancer Center, Los Angeles, CA, 90089-9601 USA.

2 Present address: Ifremer, Centre Océanologique du Pacifique, BP 7004-Taravao, 98719 Tahiti, French Polynesia.
\end{abstract}

\footnotetext{
*: Corresponding author : Evelyne Bachère, Tel.: +330467144710; fax: +330467144622 ; email address : ebachere@ifremer.fr
}

\begin{abstract}
:
Diverse families of antimicrobial peptides and proteins have been described in oysters. We investigated here how antimicrobials are involved in the immune response against a pathogenic strain of Vibrio splendidus. Oyster antimicrobials were shown to display a wide variety of expression profiles in hemocyte populations and tissues. Oyster defensins are constitutively expressed in specific tissues such as mantle $(C g$-Defm) or hemocytes ( $C g$-Defhs), while $C g$-BPI is inducible and $C g$-Prp appears down-regulated in hemocytes upon infection. The migratory behavior of hemocytes that express the different antimicrobials was found to be involved in the oyster response to a pathogenic Vibrio infection. Indeed, it contributes to colocalize several antimicrobials that were shown here to have synergistic activities. We propose that such a synergy, which was evidenced both within and between families of antimicrobials, might compensate for the low concentration of antimicrobials in oyster tissues.
\end{abstract}

\section{Highlights}

Oyster antimicrobials display different expression profiles in hemocytes and tissues. Hemocytes drive antimicrobials to tissues contributing with their colocalization. Oyster antimicrobials show synergistic activity in vitro. Synergy might compensate the low concentration of antimicrobials in oyster tissues.

Keywords : Pacific oyster ; Bivalve mollusk ; Innate immunity ; Bactericidal/permeability-increasing protein ; Proline-rich peptide ; Defensin ; Vibrio 


\section{Introduction}

Great efforts have been made over the past years to characterize the molecular processes involved in the defense reactions of the oyster Crassostrea gigas (for review see (Schmitt et al., 2011)). A particular attention has been paid to oyster antimicrobials, leading to the identification of various antimicrobial peptides (AMPs) and proteins by genomic approaches. First, a member of the defensin family ( $\mathrm{Cg}$-Defm) was characterized from the oyster mantle (Gueguen et al., 2006), then two additional defensins named $\mathrm{Cg}$-Defhs ( $\mathrm{Cg}$-Defh1 and $\mathrm{Cg}$ Defh2) were characterized from hemocytes (Gonzalez et al., 2007a). Members of the oyster defensin family are inhibitors of the peptidoglycan biosynthesis (Schmitt et al., 2010b). Both $\mathrm{Cg}$-Defm and $\mathrm{Cg}$-Defhs appeared to be constitutively expressed. Another peptide named $\mathrm{Cg}$ Prp, which belongs to the proline-rich AMP family, was also identified from hemocytes (Gueguen et al., 2009). Its mechanism of action remains unknown but it acts synergistically with Cg-Defhs. Finally, a bactericidal/permeability-increasing protein (BPI) was characterized. This membrane active protein is expressed in hemocytes in response to an immune challenge and constitutively expressed in various tissue epithelia of both challenged and unchallenged oysters (Gonzalez et al., 2007b). In a recent study, the molecular diversity of these antimicrobials was investigated (Schmitt et al., 2010a). Phylogenetic analyses gave evidence of highly variable sequence diversity among $C$. gigas antimicrobials. $C g$-Defs cluster in three distinct groups of which Cg-Defh1, Cg-Defh2 and Cg-Defm are the most representatives. In parallel, Cg-Prp presents a high number of variants with two lengths; the shorter peptide derived from that previously published (Gueguen et al., 2009) by a deletion of a conserved Proline-Arginine motif in the C-terminal region which corresponds to the putative mature peptide. Thereby, we named the original variant as long $C g$-Prp ( $C g$-lgPrp) and the new variant as short $C g$-Prp (Cg-stPrp). In contrast to both AMPs, $C g$-BPI is mostly represented by the only one sequence originally identified. Thus, $C$. gigas possesses a variety of diverse antimicrobials.

The aim of this study was to understand how antimicrobials are involved in the defense against pathogenic Vibrios. A particular attention was paid to Vibrio splendidus strain LGP32, which is associated to the massive summer mortalities of oysters (Gay et al., 2004a; Gay et al., 2004b). First, we monitored the expression and tissue colocalization of Cg-Defs, Cg-Prp and $\mathrm{Cg}$-BPI in oysters infected with $V$. splendidus LGP32. Tissues of interest were the hemocytes as circulating immune cells, gills and mantle as epithelia, and the adductor muscle which received the Vibrio injection. Second, to understand how families of antimicrobials interplay to control infections, we evaluated the synergic activities of oyster antimicrobials in vitro.

We evidenced that oyster antimicrobials display a wide variety of expression profiles in hemocyte populations and tissues, some being constitutively expressed in specific tissues such as mantle $(C g$-Defm) or hemocytes $(C g$-Defhs), others being inducible $(C g-B P l)$ or apparently down-regulated ( $C g$-Prp) in hemocytes upon infection. In addition, hemocytes were found here to serve as vehicle for given peptides like $\mathrm{Cg}$-Defhs, driving them to tissues exposed to microorganisms where other peptides like $\mathrm{Cg}$-Defm or $\mathrm{Cg}$-BPI are constitutively expressed. Finally, we showed that oyster antimicrobials exhibit distinct spectra of activity that can be greatly extended through synergy between antimicrobials and between variants of the same AMP family. This synergy likely contributes to extend oyster defense against pathogens. 


\section{Material and Methods}

\subsection{Animals, tissue collection and immune challenges}

C. gigas adult oysters were purchased from a local farm located in the Mediterranean SeaThau lagoon, Mèze, France. Oysters were kept until their use in sea water tanks at $15^{\circ} \mathrm{C}$ at the IFREMER aquaculture platform at Palavas, France. First, a small notch was carved in the dorsal side of the oyster shell, adjacent to the adductor muscle. Animals were acclimated for 5 days to let oysters recover from the stress induced by the notch. After the acclimatization period, $100 \mu \mathrm{l}$ of (i) a sub lethal dose of live virulent $V$. splendidus LGP32 $\left(1.5 \times 10^{8}\right.$ CFU/oyster) or (ii) sea water, as injury control, was injected in the oyster adductor muscle. Three groups of 10 oysters were used in each experimental condition and mortality was observed neither in controls nor in challenged oysters. Hemolymph from unchallenged and challenged oysters was collected at $12 \mathrm{~h}$ from the pericardial cavity. Hemocytes were isolated by centrifugation to discard plasma $\left(700 \mathrm{xg}\right.$ for $10 \mathrm{~min}$ at $\left.4^{\circ} \mathrm{C}\right)$.

For RNA extractions, tissues from 10 oysters were pooled; hemocyte pellets, mantles, adductor muscles and gills were resuspended in $1 \mathrm{ml}$ of TRIzol reagent (Invitrogen).

For immunofluorescence assays, hemolymph of 30 oysters was collected at $12 \mathrm{~h}$ from unchallenged and challenged oysters in antiaggregant Modified Alsever Solution MAS (9mM EDTA, $27 \mathrm{mM}$ Na-citrate, $336 \mathrm{mM} \mathrm{NaCl}, 115 \mathrm{mM}$ Glucose, $\mathrm{pH} 7$ ). Hemocytes were obtained by centrifugation $\left(700 \mathrm{xg}, 10 \mathrm{~min}, 4^{\circ} \mathrm{C}\right)$ and then fixed in MAS $4 \%$ paraformaldehyde for $10 \mathrm{~min}$ at room temperature. All experimental infections were performed according to the IFREMER animal care guideline and policy.

\subsection{RNA extraction and cDNA synthesis}

Total RNA was extracted from C. gigas hemocytes, gill, adductor muscle and mantle tissues using Trizol reagent according to manufacturer instructions (Invitrogen). RNA was then treated with DNAse I (Invitrogen), 15min at room temperature and inactivated by heat, 10min at $65^{\circ} \mathrm{C}$, followed by a second precipitation with sodium acetate $0.3 \mathrm{M}$. Then, quantification and quality of total RNA were determined using a NanoDrop spectrophotometer (NanoDrop Technologies) and agarose gel electrophoresis, respectively. Following heat denaturation of $1 \mu \mathrm{g}$ of total RNA $\left(65^{\circ} \mathrm{C}\right.$ for $\left.5 \mathrm{~min}\right)$, first strand synthesis was carried out using $50 \mathrm{ng}$ oligo(dT) ${ }_{12-18}, 1 \mathrm{mM}$ dNTPs, $1 \mathrm{U}$ RnaseOUT (Invitrogen) and 200U M-MLV reverse transcriptase in reverse transcriptase buffer (Invitrogen) following the manufacturer protocol.

\subsection{Real-time quantitative PCR (qPCR) analysis of gene expression}

Gene expression of antimicrobial effectors was analyzed by qPCR on the LightCycler 480 System (Roche) in a final volume of $6 \mu \mathrm{l}$ containing $0.5 \mu \mathrm{M}$ of each primer, $1 \mu \mathrm{l}$ of each reverse transcribed RNA (diluted at 1/8) and $5 \mu$ of qPCR reaction mix (LightCycler 480 Master 1X, Roche). Primer sequences are listed in Table 1. Universal primers were designed for each antimicrobial effectors based in the overall sequence diversity previously described (Schmitt et al., 2010a). qPCR assays were submitted to an initial denaturation step of $900 \mathrm{~s}$ at $95^{\circ} \mathrm{C}$ followed by 35 cycles of denaturation at $95^{\circ} \mathrm{C}$ for $10 \mathrm{~s}$, annealing at $57^{\circ} \mathrm{C}$ for $20 \mathrm{~s}$ and extension time at $72^{\circ} \mathrm{C}$ for $25 \mathrm{~s}$. At the end of amplification cycles, melting curves were generated to verify primer specificities. QPCR assays were performed in triplicate, and standard curves were generated using six two-fold serial dilutions from a pool of all cDNAs to determine primer pair efficiencies $(E)$ according to the equation: $E=10^{[-1 / \text { slope] }}$. Only primer pairs with efficiencies of $2 \pm 0.5$ were selected for qPCR relative quantification using the $2^{-\Delta \Delta C q}$ method (Pfaffl, 2001) with the C. gigas Elongation Factor-1 $\alpha$ as reference gene (GenBank 
ID: AB122066). Data were analyzed using LightCycler 480 software version 1.5.0.39 and the $2^{\text {nd }}$ derivative max algorithm. Statistical significance was determined using Student's $t$-test between conditions and differences were considered when $p<0.05$. Results are expressed as mean values \pm SD from 3 pools of 10 oysters by each condition.

\subsection{Recombinant expression of $\mathrm{Cg}$-Defs}

Recombinant oyster defensins $\mathrm{Cg}$-Defm, $\mathrm{Cg}$-Defh1 and $\mathrm{Cg}$-Defh2 were expressed in $\mathrm{E}$. coli Rosetta (DE3) as an N-terminal His6-tagged fusion protein using the pET-28a system (Novagen) and produced as previously described (Gueguen et al., 2006; Schmitt et al., 2010b). Amino acid sequences of variants are listed in Table 2.

\subsection{Recombinant expression of $\mathrm{Cg}-\mathrm{BPI}$}

The recombinant construction of the bactericidal/permeability increasing protein $\mathrm{Cg}$-BPI was produced in the recombinant system of Pichia pastoris, using the pPICK9 vector system (Invitrogen) as previously described (Gonzalez et al., 2007b). Purification of Cg-BPI from yeast culture supernatants was performed by a step gradient precipitation with $20 \%$ and $50 \%$ ammonium sulfate. The $50 \%$ precipitate was resuspended in $10 \mathrm{mM}$ potassium phosphate buffer $\left(\mathrm{pH} \mathrm{6)}\right.$ and subjected to dialysis in Cellu-Sep ${ }^{\circledR}$ cellulose tubular membrane (cut-off $6000-8000$ ) against $25 \mathrm{mM}$ potassium phosphate buffer $(\mathrm{pH} \mathrm{6}), 150 \mathrm{mM} \mathrm{NaCl}$, overnight at $4^{\circ} \mathrm{C}$. Then, sample was subjected to a cation exchange chromatography by carboxymethyl weak cation exchange resin (Macro-Prep ${ }^{\circledR} \mathrm{CM}$ support, Bio-Rad). Resin was added in a 1:50 ratio to the sample and incubated overnight at $4^{\circ} \mathrm{C}$ with gentle agitation. The resin was packed in a $1 \mathrm{ml}$ column and washed with 10 resin volumes of $25 \mathrm{mM}$ sodium phosphate buffer $(\mathrm{pH} \mathrm{7.3)}$ at $5 \mathrm{ml} / \mathrm{min}$. Elution was performed with 3 volumes of $25 \mathrm{mM}$ sodium phosphate buffer $(\mathrm{pH} 7.3)$ supplemented with $200 \mathrm{mM} \mathrm{NaCl}$. Protein concentration was determined using the microBCA method and protein profile was controlled by SDS-PAGE electrophoresis.

\subsection{Chemical synthesis of $\mathrm{Cg}$-Prp variants}

Chemical synthesis of $C g$-lgPrp, $C g$-stPrp and two variants representative of their diversity Cg-lgPrp P/Q and Cg-stPrp K/E (Schmitt et al., 2010a) was performed by Fmoc chemistry and purchased from GENEPEP (Montpellier, France). Amino acid sequences of peptides are listed in Table 2.

\subsection{Antimicrobial Assays}

\subsubsection{Determination of Minimal Inhibitory Concentrations}

Antimicrobial activity was assayed against several bacteria including the Gram-positive Micrococcus lysodeikticus (CIP 5345), Bacillus megaterium (CIP 6620), Staphylococcus aureus (CIP 103428), S. aureus SG511 (Sass and Bierbaum, 2009), S. haemolyticus (generous gift from P. Bulet) the marine Gram-positive bacteria Brevibacterium stationi (CIP 104228) and Microbacterium maritypicum (CIP 105733), the Gram-negative E. coli SBS363, Vibrio anguillarum (ATCC 19264), V. nigripulchritudo (CIP 103195) and oyster pathogens, V. splendidus LGP32 (CIP 107715) and V. aestuarianus LPi 02/41 (CIP 102971). Minimum inhibitory concentrations (MICs) were determined in triplicate by the liquid growth inhibition assay based on the procedure described by Hétru and Bulet (Hetru and Bulet, 1997). Poor broth (PB: $1 \%$ bactotryptone, $0.5 \% \mathrm{NaCl} w / \mathrm{v}, \mathrm{pH} 7.5$ ) nutrient medium was used for standard bacteria and artificial sea water (ASW) (Saulnier et al., 2000) supplemented with $0.4 \%$ bactopeptone and $10 \%$ yeast extract (referred to as Zobell medium) at a third strength was 
used for marine bacteria. Growth was monitored spectrophotometrically at $630 \mathrm{~nm}$ on a Multiscan microplate reader (Labsystems). MIC values are expressed as the lowest concentration tested that causes $100 \%$ of growth inhibition $(\mu \mathrm{M})$.

\subsubsection{Determination of Fractional Inhibitory Concentrations}

Synergy between 2 antimicrobial molecules was tested using the checkerboard microtiter assay (Rabel et al., 2004). To detect a possible reduction of the MIC values of each peptide when used in combination, 2-fold serial dilutions of one peptide were tested against 2-fold serial dilutions of the other peptide. Results were expressed as the fractional inhibitory concentration (FIC) index according to the following formula: $F I C=(A) / M^{\prime C} C_{A}+(B) / M C_{B}$, where $\mathrm{MIC}_{\mathrm{A}}$ and $\mathrm{MIC}_{\mathrm{B}}$ are the MICs of peptides $\mathrm{A}$ and $\mathrm{B}$ tested alone and $(\mathrm{A})$ and $(\mathrm{B})$ are the MICs of the two peptides tested in combination. FIC index were interpreted as follows: $\leq 0.5$, strong synergy; 0.5-1 synergy; $\geq 1$; additive effect; $=2$, no effect; $\geq 2$, antagonism. An FIC index of 0.5 indicates a fourfold decrease in the MIC of each compound tested, while an FIC index of 1.0 indicates a twofold decrease in the MIC of each compound tested. When MIC values are higher than the highest concentration we tested, we chose this value as the MIC in the calculation of the FIC index.

\subsection{Polyclonal antibodies and Immunofluorescence}

Immunofluorescence analyses were carried out using polyclonal antibodies specific for $\mathrm{Cg}$ Defs and $\mathrm{Cg}$-BPI. Antibodies against $\mathrm{Cg}$-Defs were raised in New-Zeland rabbits by Eurogentec (Belgium), and antibodies against $\mathrm{Cg}$-BPI were raised in Balb/C mice as described before (Gueguen et al., 2009). Hemocytes fixed with 4\% paraformaldehyde were cytocentrifuged (900rpm $5 \mathrm{~min}$ at room temperature) on L-polyLysine slides. Hemocytes were permeabilized for $10 \mathrm{~min}$ at room temperature with $0.5 \%$ Triton $\mathrm{X} 100$ in $25 \mathrm{mM}$ Tris(2,3dibromopropyl) phosphate, $50 \mathrm{mM}$ ammonium chloride, $0.2 \%$ gelatin. Two hour incubation at room temperature was performed in phosphate buffer saline (PBS) containing $5 \%$ nonfat milk to block nonspecific antibody binding. After three washes for $3 \mathrm{~min}$ at room temperature, slides were incubated $2 \mathrm{~h}$ at $37^{\circ} \mathrm{C}$ with both anti- $\mathrm{Cg}$-Def $(20 \mu \mathrm{g} / \mathrm{ml})$ and anti-Cg-BPI $(20 \mu \mathrm{g} / \mathrm{ml})$ antibodies in PBS $/ 0.5 \%$ nonfat milk. As control, irrelevant antibodies were used. After three washes in PBS, hemocytes were incubated for $1 \mathrm{~h}$ at room temperature with anti-mouse Alexa Fluor 488-conjugated (Invitrogen) and anti-rabbit Cy5-conjugated (Jackson) diluted at $1: 100$ in PBS PBS/0.5\% nonfat milk. Slides were washed three times in PBS and mounted in ProLong Gold Antifade Reagent (Invitrogen). Slides were observed by confocal microscopy Leica TCS 4D.

\section{Results}

\subsection{Expression of oyster antimicrobials after a Vibrio challenge}

The expression of $\mathrm{Cg}$-Defhs, Cg-Defm, Cg-Prp and $\mathrm{Cg}-\mathrm{BPI}$ was analyzed by quantitative PCR (qPCR) in oysters facing a microbial challenge with the pathogen Vibrio splendidus LGP32. Expression was monitored in hemocytes $12 \mathrm{~h}$ after challenge (Fig. 1A). Gills and mantle were also analyzed to detect the transcripts of interest in tissues and/or hemocytes infiltrating tissues. In our experiments, oysters received a sub lethal intramuscular injection of $V$. splendidus to obtain reproducible and standardized Vibrio infections (Duperthuy et al., 2010; Labreuche et al., 2006). Controls were (i) non-injected oysters and (ii) sterile sea-water (SSW) injected oysters for injury control. In those experiments, universal primers were used for hemocyte defensins ( $\mathrm{Cg}$-Defhs) and proline-rich peptides (Cg-Prp). Specific primers were used for $C g$-BPl, which was earlier shown to exist as a single form (Schmitt et al., 2010a). 
After the Vibrio challenge, the abundance of $C g-B P I$ transcripts increased by 2.5 -fold in circulating hemocytes $(p<0.05)$ but remained stable in other tested tissues. No significant change was observed in non-injected and injury control oysters (Fig. 1A). In contrast to $\mathrm{Cg}$ $\mathrm{BPI}$, the transcript numbers of $\mathrm{Cg}$-Defhs and $\mathrm{Cg}$-Prp decreased in circulating hemocytes after Vibrio challenge by 4 and 2.5 -fold $(p<0.05)$, respectively. Such a decrease was also observed for $\mathrm{Cg}$-Defhs upon injection of SSW (Fig. 1A). Interestingly, great abundance of $\mathrm{Cg}$-Defh transcripts were observed in the gills compared to circulating hemocytes or mantle (Fig. 1A). Neither injury nor Vibrio injection significantly modified the transcript abundance of $\mathrm{Cg}$-Defhs and $\mathrm{Cg}$-Prp in gills. In the mantle, only Cg-Prp transcripts were significantly less abundant (3-fold change, $\mathrm{p}<0.05)$ in Vibrio- than in SSW-injected oysters. Finally, Cg-Defm expression was restricted to the mantle where the number of transcripts did not change significantly in response to injury or Vibrio challenge (Fig. 1A).

Since significant and opposite variations in numbers of Cg-Defhs, Cg-Prp and Cg-BPI transcripts were observed in circulating hemocytes following challenge, we asked whether this was due to the differential migration of hemocyte populations towards infected tissues. Thus, we extended the analysis of AMP expression to the site of injection (adductor muscle). For that, circulating hemocytes and muscle tissue were analyzed for AMP expression $12 \mathrm{~h}$ after a novel injection of oysters with $V$. splendidus LGP32 (Fig. 1B). Whereas the number of $\mathrm{Cg}$-BPI transcripts increased by 3 -fold in circulating hemocytes after the Vibrio injection as previously shown (Gonzalez et al., 2007b), it remained stable at low basal levels at the injection site. On the contrary, the abundance of $C g$-Defh transcripts in circulating hemocytes decreased by 4-fold after the Vibrio challenge and concomitantly increased by 4-fold at the injection site $(p<0.05)$. Comparatively, the relative transcript abundance of $\mathrm{Cg}$-Prp in circulating hemocytes and muscle tissue was similar for non-challenged oysters, but it decreased by 3.5 and 3 -fold respectively in both tissues $(p<0.05)$ after Vibrio challenge (Fig. 1B).

\subsection{Colocalization of $\mathrm{Cg}$-BPI and $\mathrm{Cg}$-Defhs in circulating hemocytes}

The colocalization of $\mathrm{Cg}$-Defhs and Cg-Prp in circulating hemocytes was previously demonstrated (Gueguen et al., 2009). Consequently, we investigated here the colocalization of $\mathrm{Cg}$-BPI and $\mathrm{Cg}$-Defhs by immunofluorescence microscopy using specific polyclonal antibodies. $\mathrm{Cg}$-BPI was evidenced by the green fluorescence of an Alexa Fluor ${ }^{\circledR}$ 488coupled secondary antibody, while $\mathrm{Cg}$-Defs were evidenced by the red fluorescence of a Cy5-coupled secondary antibody. Cg-BPI was immunodetected in some hemocytes together with Cg-Defhs (Fig. 2). Nevertheless, in some cases, hemocytes were only positive for one antimicrobial, showing that these antimicrobials have distinct expression patterns in the different hemocyte populations. As shown in Fig. 2, strong fluorescent immunolabelling for $\mathrm{Cg}$-BPI was observed in cytoplasmic organelles or granules of hemocytes, while Cg-Defh immunolabelling was rather diffuse, being uniformly distributed throughout the cytoplasm (Fig. 2A). In addition, merged confocal microscopy photographs revealed that $\mathrm{Cg}$-Defh and $\mathrm{Cg}$-BPI fluorescence signals are at times superimposed, indicating close subcellular associations (Fig. 2B). When anti- $\mathrm{Cg}$-Def and anti-Cg-BPI antibodies were replaced by non specific antibodies, respective Alexa Fluor ${ }^{\circledR} 488$ and Cy5 fluorescence was no longer observed in any of the slides examined (data not shown).

\subsection{Spectrum of antimicrobial activities and synergistic activities of $\mathrm{Cg}$-Defs, $\mathrm{Cg}$-BPI and $\mathbf{C g}$-Prp variants}

Three recombinant variants of $C g$-Defs (mantle and hemocyte variants), four synthetic variants of $\mathrm{Cg}$-Prp (both long and short variants) and one recombinant $\mathrm{Cg}$-BPI corresponding to the single known form (Table 2) were analyzed for their antimicrobial properties. We found that every family of antimicrobials has a distinct spectrum of activity (Table 3 ). Oyster 
defensins were active at very low concentration $(0.01-6 \mu \mathrm{M})$ against most of the Grampositive bacteria tested, but did not display significant antimicrobial activity against Gramnegative bacteria $(>40 \mu \mathrm{M})$. However, oyster defensin variants displayed different potency against Gram-positive bacteria as reported earlier (Schmitt et al., 2010b). Cg-Prp variants were poorly active against the Gram-positive and Gram-negative tested bacteria, but the variants also displayed different antimicrobial potency. Thus, both $C g$-lgPrp and $C g$-lgPrp $\mathrm{P} / \mathrm{Q}$ variants were active against $M$. lysodeikticus $(80 \mu \mathrm{M})$ when only the $C g$-lgPrp variant was active against $B$. megaterium $(20 \mu \mathrm{M})$. On the counterpart, $C g$-stPrp variants did not present antimicrobial activity alone. Finally, $\mathrm{Cg}$-BPI was active at very low concentration $(0.7 \mu \mathrm{M})$ against the Gram-negative $E$. coli only and at higher concentration $(10 \mu \mathrm{M})$ against the oyster pathogen $V$. splendidus.

To gain evidence of a possible synergy between oyster antimicrobials as a result of their colocalization in oyster tissues, we measured synergistic activities in vitro between families and among variants of antimicrobials (Table 4). FIC indexes were calculated for distinct combinations of antimicrobials using the checkerboard assay. All combinations of $\mathrm{Cg}$-Def variants were synergistic against Gram-negative and Gram-positive strains $(0.37<$ FIC < 0.75), while the combination of long variants of $C g$-Prp was synergistic against Gram-positive bacteria only $(\mathrm{FIC}=0.5)$. We also noticed that $C g$-stPrp variants present lower synergistic activities compared to $C g$-lgPrp variants. Both $C g$-Defs and $C g$-Prps had synergistic activities with $\mathrm{Cg}$-BPI, as observed against the Gram-positive M. lysodeikticus CIP5345 (0.23 < FIC < $0.45)$, and Gram-negative bacteria E. coli SBS363 (0.26< FIC < 0.75), but not against $V$. splendidus LGP32 (FIC = 2). Only the combination of $\mathrm{Cg}$-Def variants was synergistic against $V$. splendidus LGP32 $(0.5<$ FIC < 0.75). In addition, we found variable synergistic potency according to the different combination of the variants. Hence, the greatest synergism was observed for the combination of (i) $\mathrm{Cg}$-Defh2 and $\mathrm{Cg}$-Defm, (ii) $\mathrm{Cg}$-Defhs and $\mathrm{Cg}$-IgPrp and (iii) $\mathrm{Cg}$-BPI and $\mathrm{Cg}$-Defs, with FIC values $\leq 0.5$ against both Gram-positive and Gramnegative bacteria, corresponding to strong synergy (i.e. at least a 4-fold decrease in the MIC value of each peptide).

\section{Discussion}

We have described here the expression, tissue localization and synergy of oyster antimicrobials upon infection with the pathogenic $V$. splendidus strain LGP32. Results showed that every antimicrobial displays a different profile of transcript abundance after Vibrio challenge. We discuss here how this appears to result from distinct transcriptional regulations and hemocyte migratory behaviors. Besides, we showed that some antimicrobials have strong synergistic activities. We discuss how this makes hemocyte migratory behavior contribute to increase oyster defenses at barrier epithelia where the organism is constantly challenged by microbes.

From this study, hemocytes were by far the tested tissue which responded more significantly to the microbial challenge. Indeed, the transcript abundance of all antimicrobials was significantly altered in circulating hemocytes, except for Cg-Defm (Fig1A and B), which was exclusively expressed in the mantle tissue as reported earlier (Gueguen et al., 2006). Cg-BPI was the only antimicrobial whose transcript abundance increased in hemocytes after challenge. This is consistent with our previous in situ hybridization data (Gonzalez et al., 2007b) and supports the up-regulation of its gene expression in this tissue. In contrast to $\mathrm{Cg}$ $\mathrm{BPI}$, both $\mathrm{Cg}$-Prps and $\mathrm{Cg}$-Defhs, showed a decrease in transcript abundance in circulating hemocytes after a Vibrio challenge. In addition, both $C g$-BPI and Cg-Prp responded to the microbial challenge but not to injury, which indicated that their transcript abundance is likely dependent on activation of the immune system by Pathogen Associated Molecular Patterns (PAMPs). Because $\mathrm{Cg}$-Defs responded similarly to a microbial and non-microbial injection, 
we concluded that injury plays a major role in the alteration of $\mathrm{Cg}$-Defh transcript abundance in circulating hemocytes.

The decrease in $C g$-Defh transcript abundance is likely related to the migration of $C g$-Defhexpressing hemocytes towards the damaged tissues, as evidenced by the concomitant increase of $\mathrm{Cg}$-Defhs transcript abundance at the injection site, in the adductor muscle (Fig.1). Such an hemocyte migration was also observed in the mussel Mytilus galloprovincialis and in the shrimp Litopenaeus vannamei, where hemocytes expressing AMPs migrated and accumulated around the bacterial injection site (Mitta et al., 2000a; Mitta et al., 2000b; Muñoz et al., 2002). This hypothesis is also supported by the increase of $\mathrm{Cg}$ Defh2 transcript abundance in C. gigas gill and mantle tissues following a bacterial challenge by immersion, both tissues being highly exposed to bacteria in this type of challenge (Gonzalez et al., 2007a). Comparatively, from our qPCR data, neither Cg-BPI nor Cg-Prpexpressing hemocytes would migrate at the injection site (Fig. 1B) suggesting different chemotactic behavior and properties among the hemocyte populations. Here, we showed by immunostaining that $\mathrm{Cg}$-Defhs and $\mathrm{Cg}$-BPI are present in different hemocytes but also inside the same hemocytes (Fig. 2). Similar results where earlier reported for $\mathrm{Cg}$-Defhs and Cg-Prp (Gueguen et al., 2009). Therefore, the differences in migratory behavior of hemocytes according to the expression of different antimicrobials suggest that hemocytes can display different chemotactic behavior potentially related to different stages of maturation.

Due to the high diversity of oyster defensins (Schmitt et al., 2010a), universal primers were chosen over specific primers for detecting the $\mathrm{Cg}$-Defh family. The highest amounts of $\mathrm{Cg}$ Defh transcripts were found in oyster gills in all tested conditions (Fig. 1). Conversely, when $\mathrm{Cg}$-Defh2-specific primers were used, the abundance of $\mathrm{Cg}$-Defh2 transcripts decreased in circulating hemocytes following microbial challenge and concomitantly increased in gills likely due to the migration of expressing hemocytes (Gonzalez et al., 2007a). The universal primers used in the present study may have amplified a new member of the defensin family constitutively expressed in gills. Such detection is consistent with the previous purification of a defensin from acidic extracts of the gills of unchallenged $C$. virginica oyster (Seo et al., 2005). Here, whether Cg-Defh transcript detection is due to infiltrating hemocytes or is gill tissue-specific deserves further investigation.

In the present study, by using qPCR, Cg-Prp transcripts were detected in circulating hemocytes from non challenged oysters and their transcript abundance dropped after Vibrio challenge but not after injury. Unlike $\mathrm{Cg}$-Defs, $\mathrm{Cg}$-Prp-expressing hemocytes were not found here to migrate to mantle, gills or at the injection site after a Vibrio challenge (Fig. 1A and B). One cannot rule out the hypothesis of a migration to other tissues such as connective tissue of the digestive gland, the gonad or the intestine that $V$. splendidus could colonize. Alternatively, Cg-Prp expression could be down-regulated in the hemocytes following the Vibrio infection. Down-regulation of AMP expression in response to a pathogenic challenge has been previously reported in vertebrate intestines (Islam et al., 2001; Salzman et al., 2003). This phenomenon, which is pathogen-driven, could obviously not be observed upon injection of heat-killed bacteria as done in previous studies (Gueguen et al., 2009). In this case, like $C g-B P I$, the modulation of $C g$-Prp expression could be associated to PAMPs.

From our study, the migratory behavior of hemocytes that express the different antimicrobials is likely a major element of the oyster response to challenge or injury, which may contribute to colocalize the antimicrobials. Indeed, by migration of antimicrobial-expressing hemocytes and continuous tissue expression, some oyster antimicrobials are likely merged in specific tissues exposed to microorganisms. One limitation of this study has been the lack of immunodetection of oyster antimicrobials in tissues other than hemocytes, which is probably indicative of their low concentration. This prevented us from colocalizing oyster antimicrobial peptides and proteins in tissues. However, this could be achieved by qPCR amplification. Indeed our qPCR experiments indicated that $\mathrm{Cg}$-Defh-expressing hemocytes infiltrate 
massively infected tissues. These tissues can express specific antimicrobials such as $\mathrm{Cg}$ Defm in the oyster mantle (Fig. 1A). The strong synergy evidenced here between Cg-Defhs and $\mathrm{Cg}$-Defm (Table 4) supports further the contribution of hemocytes in increasing oyster defenses at barrier epithelia. Besides, the colocalization of antimicrobials inside given hemocyte populations was shown here for $\mathrm{Cg}$-Defhs and $\mathrm{Cg}$-BPI (Fig. 2) and earlier for $\mathrm{Cg}$ Defhs and Cg-Prp (Gueguen et al., 2009). The synergistic activities evidenced between these distinct families of antimicrobials expressed in the same cells (Table 4) are also in strong favor of a beneficial interaction to fight infections.

That AMPs may interact simultaneously against pathogens due to their colocalization has been proposed in species other than oysters (Yeaman and Yount, 2003). Recent studies also suggest that the production of a large number of AMPs within the same animal is an evolutionary strategy to increase the spectrum of antimicrobial activities by using combinations of peptide isoforms (Mangoni and Shai, 2009). Consistently, our present study shows that through their diversity, oyster antimicrobials can fight a broad spectrum of microbes (Tables 3 and 4) while individual families have rather narrow spectra of activities (Gonzalez et al., 2007b; Gueguen et al., 2006). A number of studies have also proposed that peptide interactions may be important for overall antimicrobial activity. For example, in mammals, various antimicrobial peptides function synergistically with lysozyme in vitro (Yan and Hancock, 2001). In amphibians, synergism has also been established between several variants of the temporin AMPs (Rosenfeld et al., 2006). Similarly, together with the high diversity of oyster AMPs (Schmitt et al., 2010a), the synergistic activities evidenced here between oyster AMP variants and between families of oyster antimicrobials are likely important determinants of oyster defense.

In conclusion, through the migratory properties of oyster hemocytes expressing diverse families of antimicrobials and the basal expression of antimicrobials in tissues, synergistic activities could take place and contribute to control microbial invasion. We propose that such a synergy, which was evidenced both within and between families of antimicrobials, is essential during the oyster immune response, and that the high degree of sequence diversity in oyster antimicrobials compensates through synergic activities their low concentration in oyster tissues.

\section{Acknowledgments}

We are very grateful to Julie Fievet and Marc Leroy for precious technical assistance. This work received financial support by the Ifremer, the CNRS, the University of Montpellier 2 and the Languedoc-Roussillon Region ("chercheur(se) d'avenir" REVAResp project). Data used in this work were partly produced through molecular genetic analysis technical facilities of the IFR119 "Montpellier Environnement Biodiversité; platform qPCR Montpellier GenomiX" and through the Platform MRI DBS-UM2 Optique. Paulina Schmitt was supported by a graduate scholarship from Program Becas Chile-CONICYT.

\section{References}

Duperthuy M., Binesse J., Le Roux F., Romestand B., Caro A., Got P., Givaudan A., Mazel D., Bachère E. and Destoumieux-Garzón D., 2010. The major outer membrane protein OmpU of Vibrio splendidus contributes to host antimicrobial peptide resistance and is required for virulence in the oyster Crassostrea gigas. Environ. Microbiol. 12, 951-963. 
Gay M., Berthe F. C. and Le Roux F., 2004a. Screening of Vibrio isolates to develop an experimental infection model in the Pacific oyster Crassostrea gigas. Dis. Aquat. Organ. 59, 49-56.

Gay M., Renault T., Pons A. M. and Le Roux F., 2004b. Two Vibrio splendidus related strains collaborate to kill Crassostrea gigas: taxonomy and host alterations. Dis. Aquat. Organ. 62, 65-74.

Gonzalez M., Gueguen Y., Desserre G., de Lorgeril J., Romestand B. and Bachère E., 2007a. Molecular characterization of two isoforms of defensin from hemocytes of the oyster Crassostrea gigas. Dev. Comp. Immunol. 31, 332-9.

Gonzalez M., Gueguen Y., Destoumieux-Garzón D., Romestand B., Fievet J., Pugniere M., Roquet F., Escoubas J. M., Vandenbulcke F., Levy O., Saune L., Bulet P. and Bachère $E$., 2007b. Evidence of a bactericidal permeability increasing protein in an invertebrate, the Crassostrea gigas Cg-BPI. Proc. Natl. Acad. Sci. U S A 104, 1775964.

Gueguen Y., Herpin A., Aumelas A., Garnier J., Fievet J., Escoubas J. M., Bulet P., Gonzalez M., Lelong C., Favrel P. and Bachère E., 2006. Characterization of a defensin from the oyster Crassostrea gigas. Recombinant production, folding, solution structure, antimicrobial activities, and gene expression. J. Biol. Chem. 281, 313-23.

Gueguen Y., Romestand B., Fievet J., Schmitt P., Destoumieux-Garzón D., Vandenbulcke $F$., Bulet $P$. and Bachère $E$., 2009. Oyster hemocytes express a proline-rich peptide displaying synergistic antimicrobial activity with a defensin. Mol. Immunol. 46, 516522.

Hetru C. and Bulet P., 1997. Strategies for the isolation and characterization of antimicrobial peptides of invertebrates. Methods. Mol. Biol. 78, 35-49.

Islam D., Bandholtz L., Nilsson J., Wigzell H., Christensson B., Agerberth B. and Gudmundsson G., 2001. Downregulation of bactericidal peptides in enteric infections: a novel immune escape mechanism with bacterial DNA as a potential regulator. Nat. Med. 7, 180-5.

Labreuche Y., Lambert C., Soudant P., Boulo V., Huvet A. and Nicolas J. L., 2006. Cellular and molecular hemocyte responses of the Pacific oyster, Crassostrea gigas, following bacterial infection with Vibrio aestuarianus strain 01/32. Microbes Infect. 8, 2715-24.

Mangoni M. L. and Shai Y., 2009. Temporins and their synergism against Gram-negative bacteria and in lipopolysaccharide detoxification. Biochim. Biophys. Acta. 1788, 16109.

Mitta G., Hubert F., Dyrynda E. A., Boudry P. and Roch P., 2000a. Mytilin B and MGD2, two antimicrobial peptides of marine mussels: gene structure and expression analysis. Develop. Comp. Immunol. 24, 381-393.

Mitta G., Vandenbulcke F., Hubert F., Salzet M. and Roch P. 2000b. Involvement of mytilins in mussel antimicrobial defense. J. Biol. Chem. 275, 12954-12962.

Muñoz M., Vandenbulcke F., Saulnier D. and Bachère E., 2002. Expression and distribution of penaeidin antimicrobial peptides are regulated by haemocyte reactions in microbial challenged shrimps. Eur. J. Biochem. 269, 2678-2689.

Pfaffl M. W., 2001. A new mathematical model for relative quantification in real-time RT-PCR. Nucleic Acids Res. 29, e45.

Rabel D., Charlet M., Ehret-Sabatier L., Cavicchioli L., Cudic M., Otvos L., Jr. and Bulet P., 2004. Primary structure and in vitro antibacterial properties of the Drosophila melanogaster attacin C Pro-domain. J. Biol. Chem. 279, 14853-9.

Rosenfeld Y., Barra D., Simmaco M., Shai Y. and Mangoni M. L., 2006. A synergism between temporins toward Gram-negative bacteria overcomes resistance imposed by the lipopolysaccharide protective layer. J. Biol. Chem. 281, 28565-74.

Salzman N. H., Chou M. M., de Jong H., Liu L., Porter E. M. and Paterson Y., 2003. Enteric salmonella infection inhibits Paneth cell antimicrobial peptide expression. Infect. Immun. 71, 1109-15. 
Sass P. and Bierbaum G., 2009. Native graS mutation supports the susceptibility of Staphylococcus aureus strain SG511 to antimicrobial peptides. Int. J. Med. Microbiol. 299, 313-22.

Saulnier D., Avarre J. C., Le Moullac G., Ansquer D., Levy P. and Vonau V., 2000. Rapid and sensitive PCR detection of Vibrio penaeicida, the putative etiological agent of syndrome 93 in New Caledonia. Dis. Aquat. Organ. 40, 109-15.

Schmitt P., Duperthuy M., Montagnani C., Bachère E. and Destoumieux-Garzón D., 2011. Immune responses in the Pacific oyster Crassostrea gigas. An overview with focus on summer mortalities. In Oysters: physiology, ecological distribution and mortality (Edited by Qin J.), Vol. 1. Nova Publishers.

Schmitt P., Gueguen Y., Desmarais E., Bachère E. and de Lorgeril J., 2010a. Molecular diversity of antimicrobial effectors in the oyster Crassostrea gigas. BMC Evol. Biol. 10, 23.

Schmitt P., Wilmes M., Pugnière M., Aumelas A., Bachère E., Sahl H. G., Schneider T. and Destoumieux-Garzón D., 2010b. Insight into invertebrate defensin mechanism of action: oyster defensins inhibit peptidoglycan biosynthesis by binding to lipid II. J. Biol. Chem. 285, 29208-16.

Seo J. K., Crawford J. M., Stone K. L. and Noga E. J., 2005. Purification of a novel arthropod defensin from the American oyster, Crassostrea virginica. Biochem. Biophys. Res. Commun. 338, 1998-2004.

Yan H. and Hancock R. E., 2001. Synergistic interactions between mammalian antimicrobial defense peptides. Antimicrob. Agents. Chemother. 45, 1558-60.

Yeaman M. R. and Yount N. Y., 2003. Mechanisms of antimicrobial peptide action and resistance. Pharmacol. Rev. 55, 27-55.

\section{Tables}

Table 1. Primers used for real time PCR analysis of antimicrobial effector gene expression.

\begin{tabular}{ll}
\hline Primer name & Sequence $\left(5^{\prime}-3^{\prime}\right)$ \\
\hline$C g$-Prp $F$ & CCACCATGTTCTCTCGGAGG \\
$C g$-Prp $R$ & CTTGTAGAACGGGCTAGCAC \\
$C g$-Defm $F$ & CGGGTAACCAGTTAAAGTGC \\
$C g$-Defm $R$ & ACATCTTAACCAGAGCGTGG \\
$C g$-Defhs $F$ & GTTGTAGAGCGGGCTACTGTG \\
$C g$-Defhs $R$ & CTTGGTCAGATTCAGACTGG \\
$C g$-BPI $F$ & GATAGAAATAGGAATGGACGG \\
$C g$-BPI $R$ & GTTATAGATCCACGCTGCTCC \\
$C g$-Elf- $1 F$ & GAGCGTGAACGTGGTATCAC \\
$C g$-Elf- $1 R$ & ACAGCACAGTCAGCCTGTGA \\
\hline
\end{tabular}


Table 2. Amino acid sequences of $\mathrm{C}$. gigas antimicrobial peptides and protein used in this study. Molecular weight $(\mathrm{kDa})$ and estimated isoelectric point $(\mathrm{pl})$ of the different molecules are indicated on the right.

\begin{tabular}{|c|c|c|c|}
\hline Peptide & Amino acid sequence & $\begin{array}{l}\text { Mass } \\
(\mathrm{kDa})\end{array}$ & $\mathrm{pI}$ \\
\hline $\mathrm{Cg}$-Defh1 & GFGCPRDQYKCNSHCQSIGCRAGYCDAVTLWLRCTCTDCNGKK & 4.76 & 8.50 \\
\hline $\mathrm{Cg}$-Defh2 & GFGCPGDQYECNRHCRSIGCRAGYCDAVTLWLRCTCTGCSGKK & 4.67 & 8.51 \\
\hline $\mathrm{Cg}$-lgPrp & GPIRRPKPRPRPRPE & 1.80 & 12.18 \\
\hline $\mathrm{Cg}$-lgPrp $\mathrm{P} / \mathrm{Q}$ & GPIRRPKPRPRQRPE & 1.83 & 12.18 \\
\hline $\mathrm{Cg}$-stPrp & GPIRRPKPRPRPE & 1.55 & 12.00 \\
\hline $\mathrm{Cg}$-stPrp K/E & GPIRRPEPRPRPE & 1.55 & 11.52 \\
\hline $\mathrm{Cg}$-BPI & $\begin{array}{l}\text { KTPGLQTRITDRALEYATEVALDILSKQVTGQQIPDQHGQSGDVKFDITGMNVKQFTKPSSRVSLIQNVGLSWSTSGTGLAIHG } \\
\text { DFKYKYRKGIIKISDHGSFDLKANGINFQIKIEIGMDGTGRPTMKAVGCSCNVGSADIKFHGGAAWIYNLFSGQLENKLKDMV } \\
\text { GGGNGLLCKQLNTLIDVNGMKSLQKLÒKTPGLQTRITDRALEYATEVALDILSKQVTGQQIPDQHGQSGDVKFDITGMNVKQF } \\
\text { TKPSSRVSLIQNVGLSWSTSGTGLAIHGDFKYKYRKGKTPGLQTRITDRALEYATEVALDILSKQVTGQQIPDQHGQSGDVK } \\
\text { FDITGMNVKQFTKPSSRVSLIQNVGLSWSTSGTGLAIHGDFKYKYRKGIIKISDHGSFDLKANGINFQIKIEIGMDGTGRPTMK } \\
\text { AVGCSCNVGSADIKFHGGAAWIYNLFSGQLENKLKDMVG }\end{array}$ & 50.14 & 9.37 \\
\hline
\end{tabular}

Table 3. Antimicrobial activity spectra for C. gigas antimicrobial effectors Cg-Defs, Cg-Prps and $\mathrm{Cg}$-BPI against selected bacteria. MIC (IM) values refer to the minimal inhibitory concentration required to achieve $100 \%$ growth inhibition. MICs are presented as average values from three independent experiments. Nt: not tested.

\begin{tabular}{|c|c|c|c|c|c|c|c|c|}
\hline $\operatorname{MIC}[\mu \mathrm{M}]$ & Gg-Defh2 & Cg-Defm & Cg-Defh1 & Cg-lgPrp & Cg-lgPrp P/Q & Cg-stPrp & Cg-stPrp K/E & $\mathrm{Cg}$-BPI \\
\hline \multicolumn{9}{|l|}{ Gram-positive } \\
\hline M lysodeikticus CIP5345 & 0.01 & 0.01 & 0.03 & 80 & 80 & $>80$ & $>80$ & $>20$ \\
\hline B. megaterium CIP 6620 & 0.03 & 0.03 & 0.06 & 20 & $>20$ & $>20$ & $>20$ & Nt \\
\hline S. aureus CIP 103428 & 0.25 & 2 & 2 & $>20$ & $>20$ & $>20$ & $>20$ & Nt \\
\hline S. aureus SG511 & 0.12 & 0.25 & 0.5 & $>20$ & $>20$ & $>20$ & $>20$ & Nt \\
\hline M maritypicum CIP 105733T & 1 & 1 & 2 & $>20$ & $>20$ & $>20$ & $>20$ & Nt \\
\hline R stationis CIP 101282 & 0.1 & 0.2 & 4 & $>20$ & $>20$ & $>20$ & $>20$ & Nt \\
\hline S. haemolyticus & 2 & 2 & 6 & $>20$ & $>20$ & $>20$ & $>20$ & $\mathrm{Nt}$ \\
\hline \multicolumn{9}{|l|}{ Gram-negative } \\
\hline V. splendidus LGP32 CIP 107715 & $>40$ & $>40$ & $>40$ & $>40$ & $>40$ & $>40$ & $>40$ & 10 \\
\hline V. aestuarianus & $>40$ & $>40$ & $>40$ & $\mathrm{Nt}$ & $\mathrm{Nt}$ & Nt & $\mathrm{Nt}$ & Nt \\
\hline V. anguillarum ATCC 19264 & $>40$ & $>40$ & $>40$ & Nt & $\mathrm{Nt}$ & Nt & Nt & $\mathrm{Nt}$ \\
\hline$E$ coli SBS 363 & 20 & 20 & 40 & $>80$ & $>80$ & $>80$ & $>80$ & 0.7 \\
\hline V. nigripulchritudo CIP103195 & $>40$ & $>40$ & $>40$ & $>40$ & $>40$ & $>40$ & $>40$ & $\mathrm{Nt}$ \\
\hline
\end{tabular}


Table 4. FIC index for $\mathrm{C}$. gigas antimicrobial effectors Cg-Defs, $\mathrm{Cg}$-Prps and Cg-BPI against selected bacteria.

\begin{tabular}{|c|c|c|c|}
\hline \multirow[t]{2}{*}{ FIC Index } & \multirow{2}{*}{$\begin{array}{l}\text { Gram-positive bacteria } \\
\text { M. lysodeikticus } \\
\text { CIP5345 }\end{array}$} & \multicolumn{2}{|c|}{ Gram-negative bacteria } \\
\hline & & $\begin{array}{l}\text { E. coli } \\
\text { SBS } 363\end{array}$ & $\begin{array}{l}\text { V. splendidus } \\
\text { CIP } 107715\end{array}$ \\
\hline \multicolumn{4}{|c|}{ Between Cg-Defs variants } \\
\hline Gg-Defh1 $+\mathrm{Cg}$-Defh2 & 0.50 & 0.75 & 0.75 \\
\hline G-Defh1 + Cg-Defm & 0.75 & 0.75 & 0.75 \\
\hline Qg-Defh2 + Cg-Defm & 0.37 & 0.50 & 0.50 \\
\hline \multicolumn{4}{|l|}{ Between Cg-Prp variants } \\
\hline$C g-\operatorname{lgPrp}+C g$-lgPrp P/Q & 0.50 & 2 & 2 \\
\hline G--lgPrp + Cg-stPrp & 2 & 2 & 2 \\
\hline Cg-lgPrp + Cg-stPrp K/E & 2 & 2 & 2 \\
\hline \multicolumn{4}{|c|}{ Between Cg-Defs and Cg-Prip 2236} \\
\hline G-Defh1 + Cg-lgPrp & 0.35 & 0.50 & 2 \\
\hline G-Defh $2+C g$-lgPrp & 0.30 & 0.28 & 2 \\
\hline Qg-Defm + Cg-lgPrp & 0.45 & 0.75 & 2 \\
\hline \multicolumn{4}{|c|}{ Between Cg-BPI and AMPs } \\
\hline $\mathrm{Gg}-\mathrm{BPI}+\mathrm{Gg}-\lg \mathrm{Prp}$ & $\mathrm{Nt}$ & 0.50 & \\
\hline $\mathrm{Qg}-\mathrm{BPI}+\mathrm{Gg}$-lgPrp P/Q & Nt & 0.75 & 2 \\
\hline G-BPI + Gg-stPrp & $\mathrm{Ne}$ & 0.75 & 2 \\
\hline G $\mathrm{g}-\mathrm{BPI}+\mathrm{Gg}$-stPTp K/E & $\mathrm{Nt}$ & & 2 \\
\hline G-BPI + Gg-Defh 1 & 0.45 & 0.26 & 2 \\
\hline Gg-BPI + Gg-Defh2 & 0.23 & 0.27 & 2 \\
\hline G-BPI + G-Defm & 0.23 & 0.31 & 2 \\
\hline
\end{tabular}




\section{Figures}

Figure 1. qPCR analysis of the expression of antimicrobials in the oyster $C$. gigas after a Vibrio challenge. Relative expression of $C g$-BPI, Cg-Prp, Cg-Defm and Cg-Defhs transcripts were normalized with the gene expression of $C$. gigas Elongation Factor $-1 \alpha$ and calculated using the method described by Pfaffl (Pfaffl, 2001). Results are expressed as mean values \pm SD from 3 pools of 10 oysters by each condition. A. Relative expression in circulating hemocytes, gill and mantle tissues in non stimulated oysters (c, white bars), injury control oysters (sw, striped bars) and Vibrio challenged oysters (Vs, black bars). B. Relative expression in circulating hemocytes and site of injection in non stimulated oysters (white bars) and Vibrio challenged oysters (gray bars). Asterisks indicate significant differences $(p<0.05)$.
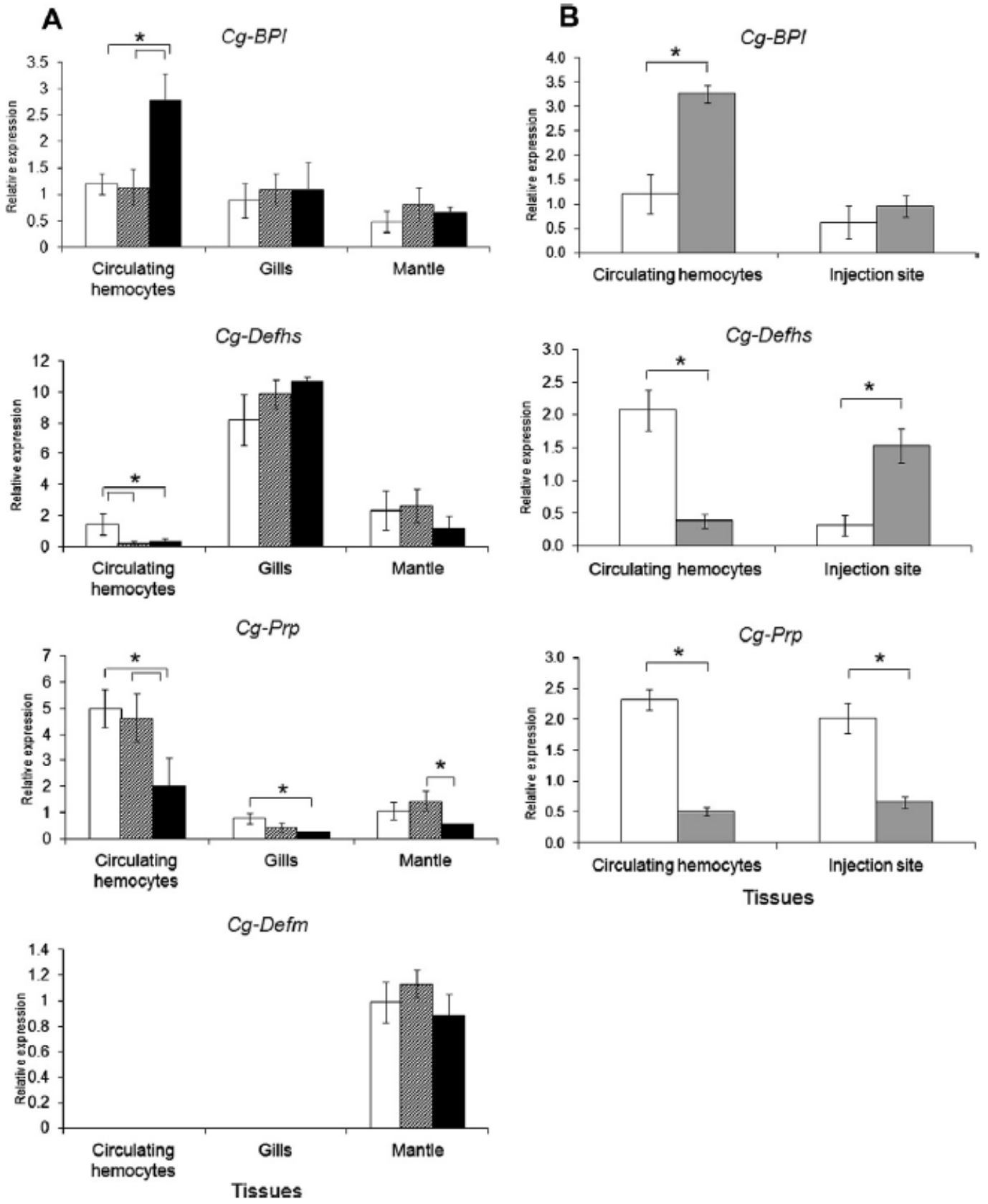
Figure 2. Detection of $\mathrm{Cg}$-BPI and $\mathrm{Cg}$-Defhs in circulating hemocytes of $C$. gigas by immunofluorescence. Cy5 staining (green) was used for $\mathrm{Cg}$-BPI detection, and Texas Red staining (red) for $\mathrm{Cg}$-Defs detection. Immunostained cells were photographed under epifluorescence confocal microscopy. A. Differences in immunolabelling patterns between $\mathrm{Cg}$-BPI and $\mathrm{Cg}$-Defhs. B. Colocalization of $\mathrm{Cg}$-BPI and $\mathrm{Cg}$-Defhs in the same subcellular locations. Arrows indicate hemocytes expressing only $\mathrm{Cg}$-BPI (A) or Cg-Defhs (B). White bars: $5 \mu \mathrm{m}$
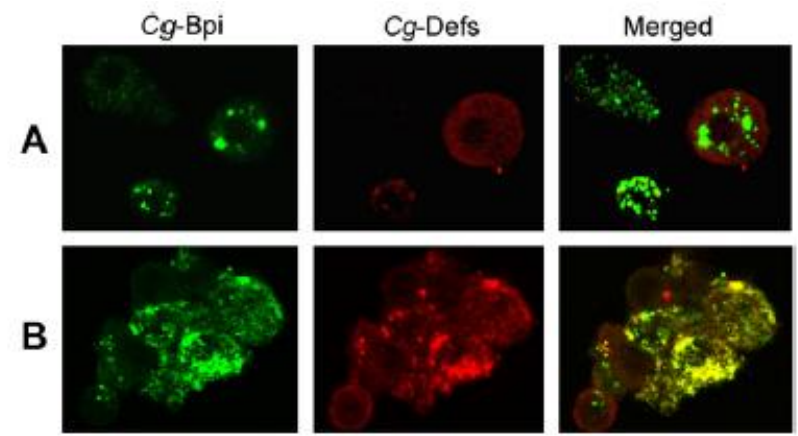